\title{
Severe Osteoarthritis of the Knee as an Early Symptom of Alkaptonuria: A Case Report
}

\author{
Alois Franz, and Mohammed Abusafieh", \\ ${ }^{1}$ Department of Orthopedics, Trauma and Hand Surgery, St. Marien Hospital, Siegen, Germany \\ ${ }^{*}$ Corresponding author: Mohammed Abusafieh, Department of Orthopedics, Trauma and Hand Surgery, St. Marien Hospital, Siegen, Germany. Tel: +49-2712311702, E-mail: \\ dr_abusafieh@yahoo.com
}

Received 2015 October 30; Revised 2015 December 13; Accepted 2015 December 25.

\begin{abstract}
Introduction: Alkaptonuria (AKU) is a very rare disease and occurs due to the deficiency of the enzyme homogentisate1, 2-dioxygenase (HGD) which results in ochronosis, a term used to describe the dark pigmentation of the tissues. Alkaptonuria affects mostly the knee joint followed by the hip as major weight bearing joints, where the life quality of such patients is severely affected.

Case Presentation: We present a case of Caucasian male who was suffering from severe osteoarthritis of the knee. Initially he underwent an arthroscopic partial meniscectomy in a nearby hospital. Fourteen months later we performed a total knee replacement using the Journey II CR prosthesis. We found a dark pigmentation of the inside of the knee (synovial tissue and cartilage). A positive urine test and a pathological specimen examination revealed the diagnosis of AKU. The patient had no complications after the surgery and underwent a standard rehabilitation program.

Conclusions: Osteoarthritis could be the first manifestation of patients with AKU and the onset of the joint disease could be lateand rapid.

Keywords: Alkaptonuria, Ochronosis, Knee Arthroplasty, Journey II CR
\end{abstract}

\section{Introduction}

Alkaptonuria (AKU) is a very rare disease. It is considered to be an autosomal recessive metabolic condition (1), which is estimated to be in a frequency range from 1:250,000 live births in the USA (2) to 1:19,000 in Slovakia (3). It occurs due to the deficiency of the enzyme homogentisate1, 2-dioxygenase (HGD) (2) which is responsible for the degradation of homogentisic acid (HGA), an intermediate product from metabolism of the amino acids tyrosine and phenylalanine, resulting in the accumulation of HGA and its metabolites in the tissues causing ochronosis (a term used to describe the dark pigmentation of tissues) (4). Symptoms of AKU include the appearance of dark urine, ear and scleral pigmentation, arthropathy of major joints and spine, calcification of cartilaginous tissue as well as deterioration of cardiac valves $(2,4)$. Most of these symptoms are observed starting in the fourth or fifth decade of life (5), except for the appearance of dark urine, which is detected during childhood, resulting from excretion and oxidation of homogentisic acid. The pigmentation also affects the tendons and ligaments, because of their high collagen content, and it causes inflammatory alterations that may lead to spontaneous tearing (6). Alkaptonuria affects mostly the knee joint followed by the hip as major weight bearing joints $(7,8)$. Arthroscopic debridement and drilling may be helpful in the early stage of ochronitic arthropathy, but most of the patients will eventually require joint replacement (9).
Although there is no proven link between the presence of homogentisic acid and shortened lifespan, the quality of life is severely affected (10). The current treatments that give proven clinical benefits are either liver or renal transplantation or more commonly joint replacement surgery $(4,11)$.

\section{Case Presentation}

A 55-year-old Caucasian male was referred to our hospital suffering from right sided knee pain for almost 2 years. The pain used to be related to movement and weight bearing and lately also in sleeping and at resting. In the last 2 years the patient was not able to practice his job as a welder. Fourteen months earlier he underwent, in a nearby hospital, an arthroscopic knee debridement and partial meniscectomy. He was diagnosed to be having a severe osteoarthritis of the right knee. There was no documentation of a dark pigmentation of the inside of the knee. The patient is known to be having severe osteoarthritis of the right shoulder, chronic hepatitis $\mathrm{C}$ infection, status after a stroke with hemiparesis of the left side with residual lightly impaired superficial sensation of the arm. The clinical examination revealed that the patient is in a good general and nutritional status. He showed a slightly painful limping on the right side with a mild varus deformity. The right knee showed two inconspicuously arthroscopy

Copyright ( ) 2016, Iran University of Medical Sciences. This is an open-access article distributed under the terms of the Creative Commons Attribution-NonCommercial 4.0 International License (http://creativecommons.org/licenses/by-nc/4.0/) which permits copy and redistribute the material just in noncommercial usages, provided the original work is properly cited. 
scars. There was mild effusion, no warming or redness of the skin. There was tenderness on palpation on the medial joint space and the patella. The range of movement was limited with a maximal flexion of 100 degrees and an extension lag of 10 degrees. The medial collateral ligament was slightly contracted, but there was no ligament insufficiency. The x-rays of the right knee in two planes in standing and the sky-view of the patella revealed an advanced joint space narrowing and osteophytes on the medial side and behind the patella (Figure 1). Due to the clinical and radiological as well as the arthroscopic findings, the patient was scheduled for a total knee replacement (TKR). The surgery was performed under general anesthesia with additional epidural analgesia. After exploring the knee, we surprisingly found a severely black pigmentation of the synovial tissue, menisci, cruciate ligaments as well as the quadriceps and patellar ligaments. The joint cartilage showed mild black pigmentation. Many specimens of the black tissues were taken for further histopathological examination. We performed a cemented TKR using the Journey II CR system (Smith and Nephew, Memphis, USA) (Figure 2). The Journey II CR is a new prosthesis using the OXINIUM technology (12) to be used in high demanding active patients aiming to preserve the anatomical 3 degrees medial inclination "varus" of the joint line (Figure 3). The patient underwent the scheduled early in-hospital mobilization on two crutches on the first postoperative day. He was discharged after ten days to start a special rehabilitation program for four weeks. The pathological consultation of the tissue specimens revealed an ochonrotic Alkaptonuria. A later performed urine test confirmed the diagnosis (Figure 4). Scheduled clinical and radiological assessments after 6 and 12 weeks revealed a satisfied patient with a good knee function with a maximal flexion of 120 degrees without any extension deficiency. The patient was advised to undergo further physiotherapy to optimize the range of movement and strengthen the thigh muscles.

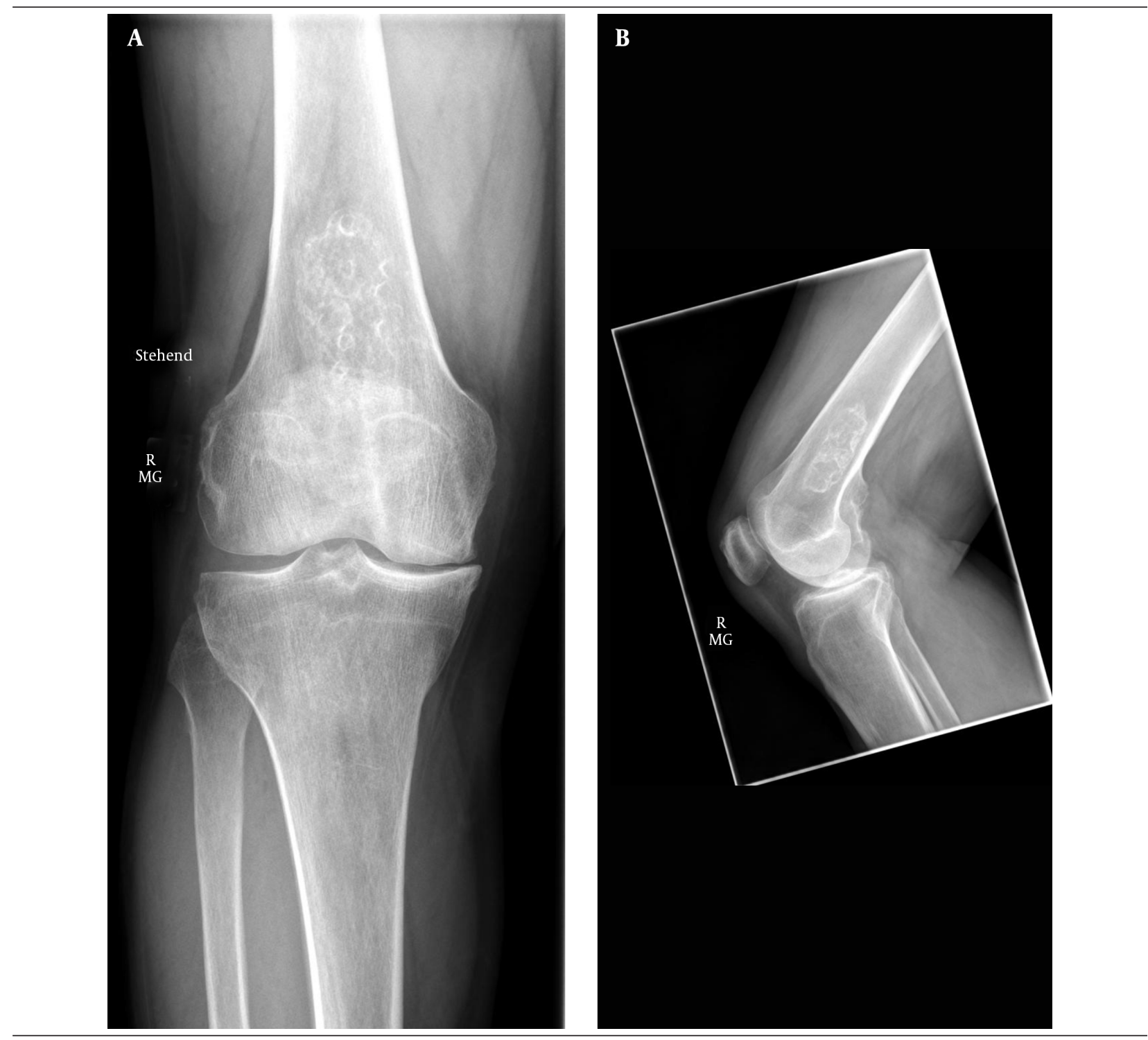

Figure 1. Preoperative ape and lateral Radiographs of the Right Knee Showing a Severe Osteoarthritis Mainly on the Medial Side 

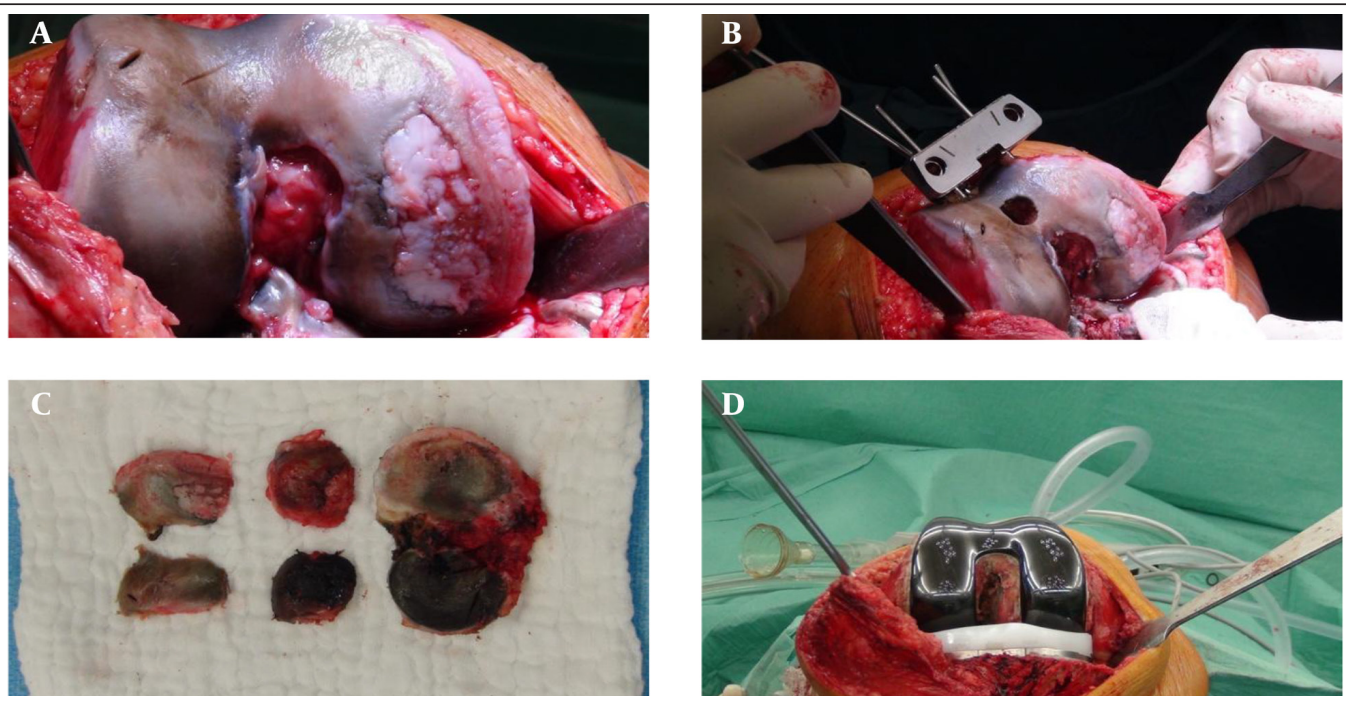

Figure 2. Intraoperative Images Showing the Black Cartilage and Soft Tissue, Affecting Mailny the Meniscus and the Synovial Tissue, and Finally Implanted Journey II CR Prothesis (OXINIUM)

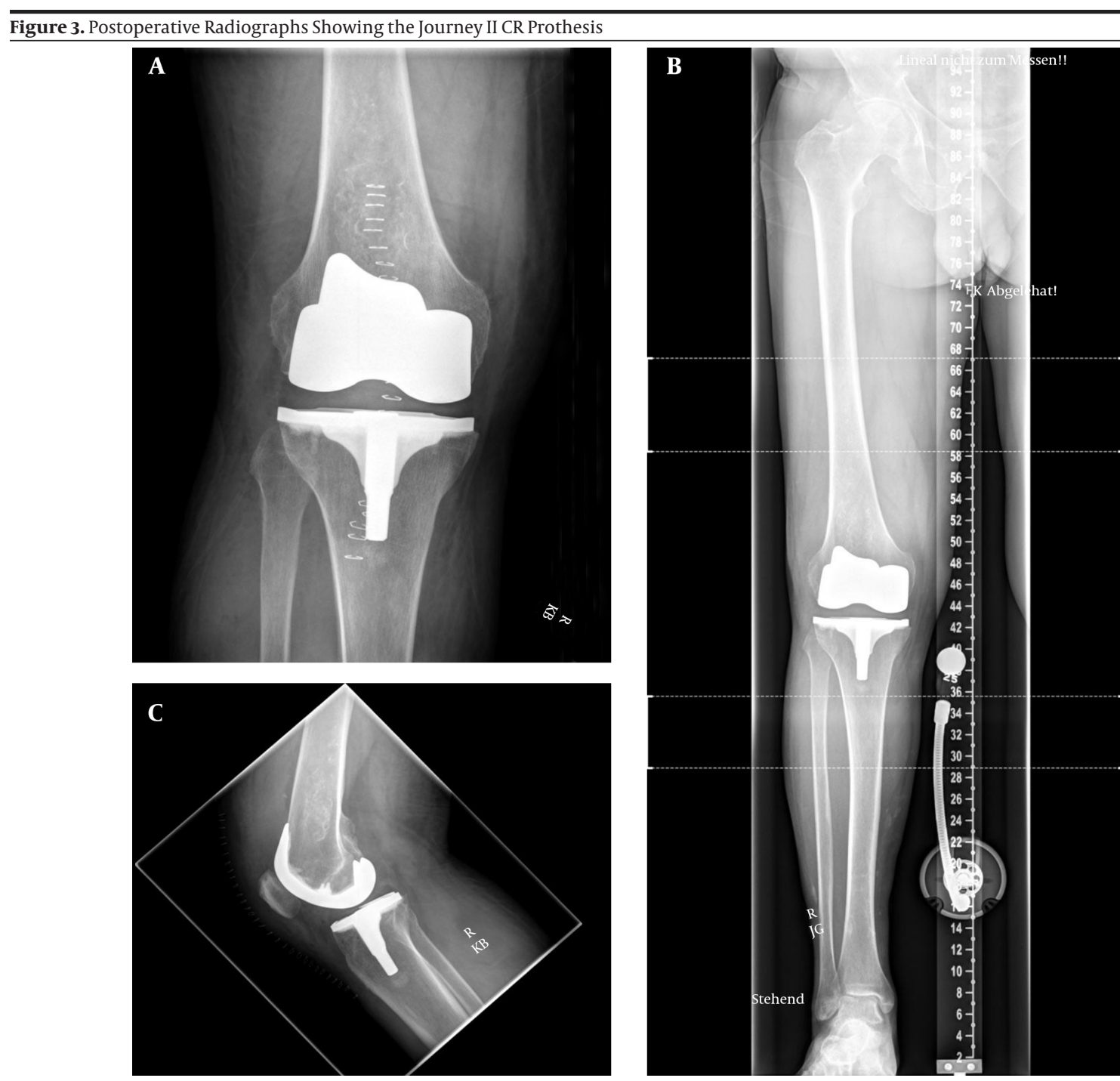

The 3 degrees of medial inclination of the joint line (varus) is achieved through the PE-insert and to be seen in the ape-radiograph. 


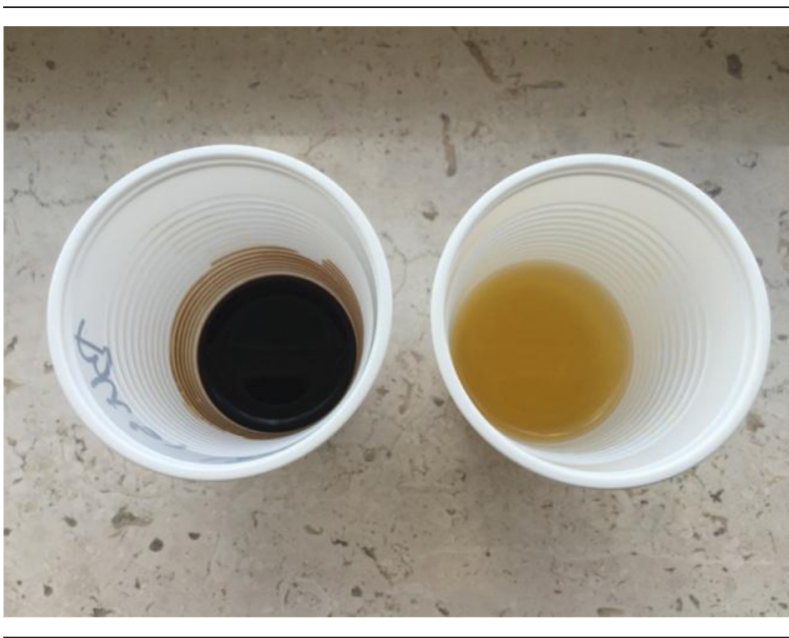

Figure 4. Positive Urine Test

\section{Discussion}

Alkaptonuria is a well-known non-malignant disease and was first described in 1584 in children with dark urine. The review of the literature showed few cases of the ochronotic joint disease. These cases are mostly presented in the form of case reports, and mostly they are from Turkey, Iran and India (13-15). Our patient has a Kazakh descent. At our hospital we perform around 1,200 arthroplasties per year. The presented case was the first one to be documented in our hospital at least in the last 10 years with an ochronotic joint disease. Early manifestations of AKU, such as dark urine are usually noticed by mothers when changing the diaper of a newborn (16). Physical symptoms of AKU, particularly ochronotic ear and scleral pigmentations, always appear after 30 years of age with progressive arthritic and organ changes as age advances $(2,4)$. Kumar et al. (17) presented 3 patients with 4 spontaneous ruptures of the patellar tendon and tendo Achilles as the first symptom of AKU. Some other cases of AKU are presented with spinal affection with major neurological and/or psychological symptoms (18). Our patient had no major peripheral symptoms of AKU other than the joint disease of the knee and probably of the shoulder. The black pigmentation of the knee joint was for us an extremely unexpected surgical finding. In the presented case, an arthroscopic debridement of the affected knee was performed in a nearby hospital 14 months earlier. In the arthroscopy, there were no hints about a tissue dark coloration, which can be a clue for a late onset and progressivity of the disease in this patient. Currently, there is no specific medical treatment for AKU and therefore the therapeutic approach is always symptomatic. However, total joint replacement in published cases of ochronotic osteoarthritis report good results similar to osteoarthritis patients without ochronosis (14). Nevertheless, there is no safe information in the literature about the type of the prosthesis. According to the literature data and to our case, the diagnosis of an ochronotic joint disease is usual- ly established during the surgery. In the most published cases there were no difficulties to perform a TKR. Spencer et al. (19) reported one case in which the patellar tendon was stiff and attenuated, making patellar dislocation difficult. In the presented case there was a strong pigmentation of the quadriceps and patellar tendons, but they were not calcified and therefore we did not have any difficulty to dislocate the patella during the surgery. This case is presented to add it to the short list of this disease in the literature. The aim is to show that in some cases, the ochronotic joint disease could be the first manifest symptom of AKU. Until now there is no evidence that osteoarthritis on top of ochronosis has worse prognosis than primary osteoarthritis (20). Therefore, we believe that the therapeutic approach in both conditions is the same and surgeons should not hesitate to perform a one-set arthroplasty of a severely damaged black joint. From the current case report, it is concluded that (1) osteoarthritis could be the first manifestation of patients with AKU, (2) the onset of the joint disease could be late and rapid, and (3) in our opinion, the joint replacement surgery might be the best intervention in such patients.

\section{References}

1. Oexle K, Engel K, Tinschert S, Haas D, Lee-Kirsch MA. Three-generational alkaptonuria in a non-consanguineous family. J Inherit Metab Dis. 2008;31 Suppl 2:S425-30. doi: 10.1007/s10545-0080994-7. [PubMed: 19096913]

2. Ranganath LR, Jarvis JC, Gallagher JA. Recent advances in management of alkaptonuria (invited review; best practice article). JClin Pathol. 2013;66(5):367-73. doi:10.1136/jclinpath-2012-200877. [PubMed: 23486607]

3. Zatkova A, de Bernabe DB, Polakova H, Zvarik M, Ferakova E, Bosak $\mathrm{V}$, et al. High frequency of alkaptonuria in Slovakia: evidence for the appearance of multiple mutations in HGO involving different mutational hot spots. Am J Hum Genet. 2000;67(5):1333-9. doi:10.1016/S0002-9297(07)62964-4. [PubMed:11017803]

4. Phornphutkul C, Introne WJ, Perry MB, Bernardini I, Murphey MD, Fitzpatrick DL, et al. Natural history of alkaptonuria. N Eng J Med. 2002;347(26):2111-21. doi: 10.1056/NEJMoa021736. [PubMed: 12501223]

5. Albers SE, Brozena SJ, Glass LF, Fenske NA. Alkaptonuria and ochronosis: case report and review. J Am Acad Dermatol. 1992;27(4):609-14. [PubMed:1401313]

6. Mannoni A, Selvi E, Lorenzini S, Giorgi M, Airo P, Cammelli D, et al. Alkaptonuria, ochronosis, and ochronotic arthropathy. Semin Arthritis Rheum. 2004;33(4):239-48. [PubMed:14978662]

7. Gaines JJ. The pathology of alkaptonuric ochronosis. Hum Pathol. 1989;20(1):40-6. [PubMed: 2643557]

8. La Du BJ. Alcaptonuria and ochronotic arthritis. Mol Biol Med 1991;8(1):31-8. [PubMed:1943688]

9. Aydogdu S, Cullu E, Ozsoy MH, Sur H. Cementless total knee arthroplasty in ochronotic arthropathy: a case report with a 4-year follow-up. J Arthroplasty. 2000;15(4):539-43. doi: 10.1054/ arth.2000.4228. [PubMed:10884219]

10. Kobak AC, Oder G, Kobak S, Argin M, Inal V. Ochronotic arthropathy: disappearance of alkaptonuria after liver transplantation for hepatitis B-related cirrhosis. J Clin Rheumatol. 2005;11(6):3235. [PubMed:16371803]

11. Introne WJ, Phornphutkul C, Bernardini I, McLaughlin K, Fitzpatrick D, Gahl WA. Exacerbation of the ochronosis of alkaptonuria due to renal insufficiency and improvement after renal transplantation. Mol Genet Metab. 2002;77(1-2):136-42. [PubMed: 12359141]

12. Hunter G, Long M. Abrasive Wear of Oxidized Zr-2.5 Nb, CoCrMo, 
and Ti-6Al-4V Against Bone Cement. 6th World Biomaterials Cong. Trans., Society for Biomaterials, Minneapolis, MN. 2000:835.

13. Sahoo MM, Mahapatra SK, Sethi GC, Dash SK. Patellar ligament rupture during total knee arthroplasty in an ochronotic patient. Acta Orthop Traumatol Turc. 2014;48(3):367-70. doi: 10.3944/ AOTT.2014.3245. [PubMed: 24901931]

14. Ozmanevra R, Guran O, Karatosun V, Gunal I. Total knee arthroplasty in ochronosis: a case report and critical review of the literature. Eklem Hastalik Cerrahisi. 2013;24(3):169-72. doi: 10.5606| ehc.2013.36. [PubMed: 24191883]

15. Siavashi B, Zehtab MJ, Pendar E. Ochronosis of hip joint; a case report. Cases J. 2009;2:9337. doi: 10.1186/1757-1626-2-9337. [PubMed: 20062596]

16. Vijaikumar M, Thappa DM, Srikanth S, Sethuraman G, Nadarajan S. Alkaptonuric ochronosis presenting as palmoplantar pigmen- tation. Clin Exp Dermatol. 2000;25(4):305-7. [PubMed: 10971492]

17. Kumar RVM, Rajasekaran S. Spontaneous tendon ruptures in alkaptonuria. Journal of Bone \& Joint Surgery, British Volume. 2003;85(6):883-6.

18. Rana AQ, Saeed U, Abdullah I. Alkaptonuria, more than just a mere disease. Journal of neurosciences in rural practice. 2015;6(2):257. [PubMed:25883496]

19. Spencer JM, Gibbons CL, Sharp RJ, Carr AJ, Athanasou NA. Arthroplasty for ochronotic arthritis: no failure of 11 replacements in 3 patients followed 6-12 years. Acta Orthop Scand. 2004;75(3):355-8. doi:10.1080/00016470410001321. [PubMed: 15260431]

20. Araki K, Sudo A, Hasegawa M, Uchida A. Devastating ochronotic arthropathy with successful bilateral hip and knee arthroplasties. J Clin Rheumatol. 2009;15(3):138-40. doi: 10.1097| RHU.0b013e31819e6b41. [PubMed:19300282] 